\title{
Three-dimensional transoesophageal echocardiography in a case of anomalous origin of the left coronary artery from the opposite sinus of Valsalva.
}

\author{
Giuliano Giusti ${ }^{1}$, Carmelo Arcidiacono ${ }^{1}$, and Marco Pozzi ${ }^{2}$ \\ ${ }^{1}$ Istituto Giannina Gaslini Istituto Pediatrico di Ricovero e Cura a Carattere Scientifico \\ ${ }^{2}$ Affiliation not available
}

April 12, 2021

\begin{abstract}
Abnormal ostial morphology and acute take-off are considered potential mechanisms of flow obstruction in the anomalous coronary artery. We present a proof of concept describing how three-dimensional transoesophageal echocardiography can be used to visualise the coronary ostium and proximal course in patients with anomalous coronary artery.
\end{abstract}

Title :

Three-dimensional transoesophageal echocardiography in a case of anomalous origin of the left coronary artery from the opposite sinus of Valsalva.

Authors:

Giuliano Giusti, MD 1, Carmelo Arcidiacono, MD 1, Marco Pozzi, MD, PhD 2.

\section{Affiliations :}

1 Unit of Pediatric Cardiology, Gaslini Pediatric Hospital, Genoa, Italy.

2 Department of Paediatric and Congenital Cardiac Surgery and Cardiology, Ospedali Riuniti, Ancona, Italy.

\section{Correspondence:}

Giuliano Giusti, MD, Unit of Paediatric Cardiology, Istituto Giannina Gaslini, Via Gerolamo Gaslini, 5, 16147, Genoa, ITALY. Email:giulianogiusti@gaslini.org. Telephone: +39-01056361. Fax number: None.

\section{Abstract:}

Abnormal ostial morphology and acute take-off are considered potential mechanisms of flow obstruction in the anomalous coronary artery. We present a proof of concept describing how three-dimensional transoesophageal echocardiography can be used to visualise the coronary ostium and proximal course in patients with anomalous coronary artery.

\section{Keywords:}

Anomalous origin of the left coronary artery from the opposite sinus of Valsalva; Anomalous Coronary Artery; three-dimensional transoesophageal echocardiography; coronary ostial morphology.

Text : 
A 43 year-old Caucasian male was admitted to our emergency department for typical chest pain occurring after exercise. The physical examination and rest electrocardiography were unremarkable. The patient was a chronic smoker with a history of untreated arterial hypertension. A coronary CT scan revealed anomalous origin of the left coronary artery from the opposite sinus of Valsalva with an inter-arterial course, without significant coronary artery disease (Figure 1). We performed a complete evaluation of the coronary anatomy with transesophageal echocardiography (Figure 2). We analyzed specific aspects of the anatomy of the anomalous coronary artery as described in recent publications.[1,2] In particular: 1) Proximal course of the coronary arteries 2) Number of ostia and ostial morphology 3) angle of take-off and 4) level of take-off of the anomalous coronary artery. Through coronary CT and transesophageal echocardiography, we detected: 1) anomalous origin of the left coronary artery from the opposite sinus with an inter-arterial course 2) A common coronary ostium in the right sinus of Valsalva, giving rise to the right and left main coronary arteries, with three- dimensional visualization 3) Acute take-off angle of the anomalous left coronary artery (defined as less than $45^{\circ}$ ) with 4 ) take-off level below the commissure.

Anomalous origin of the left coronary artery from the opposite sinus of Valsalva is an important cause of sudden death in young adults[3]. Post mortem studies have described associated acute take-off angle and slit-like elliptical ostium in the anomalous coronary artery leading to sudden death in such patients[4]. Abnormal ostial morphology and acute take-off are considered potential mechanisms of flow obstruction in the anomalous coronary artery[5]. Cross-sectional coronary angiography with cardiac CT scan or magnetic resonance is considered necessary to accomplish $3 \mathrm{D}$ visualization of the coronary artery ostium[1]. We present a proof of concept describing how three-dimensional transoesophageal echocardiography can be used to visualise the coronary ostium and proximal course in patients with anomalous coronary artery. However, the accuracy of such imaging modality in the routine evaluation of the anomalous coronary artery is unknown, and further investigation is needed.

\section{Acknowledgements: None.}

Financial Support: This research received no specific grant from any funding agency, commercial or notfor-profit sectors.

\section{Conflict of Interest: None}

Author contributions: G.G. designed and directed the project. M.P. helped supervise the project. G.G. wrote the manuscript. C.A. and M.P. authors contributed to the final version of the manuscript.

\section{References:}

1. Harris MA, Whitehead KK, Shin DC, et al. Identifying Abnormal Ostial Morphology in Anomalous Aortic Origin of a Coronary Artery. Ann Thorac Surg. Elsevier; 2015;100:174-9.

2. Cheezum MK, Liberthson RR, Shah NR, et al. Anomalous Aortic Origin of a Coronary Artery From the Inappropriate Sinus of Valsalva. J Am Coll Cardiol. 2017;69:1592-608.

3. Recruits M. Annals of Internal Medicine Article Sudden Death in Young Adults : A 25-Year Review of Autopsies in. Ann Intern Med. 2004;141.

4. Frescura C, Basso C, Thiene G, et al. Anomalous origin of coronary arteries and risk of sudden death: A study based on an autopsy population of congenital heart disease. Hum Pathol. 1998;29:689-95.

5. Mosca RS, Phoon CKL. Anomalous Aortic Origin of a Coronary Artery Is Not Always a Surgical Disease. Semin Thorac Cardiovasc Surg Pediatr Card Surg Annu. 2016;19:30-6.

\section{Legends:}

\section{Figure 1:}

Coronary CT identified features of the anomalous coronary artery in the presented case. A : The vessel's take-off level (below the commissure) shown in three - dimensional rendered images. B : Multiplanar axial 
$\mathrm{CT}$ reconstruction at the level of the coronary artery origin demonstrates the left coronary artery coursing between the aorta and the pulmonary artery. $\mathbf{C}-\mathbf{D}$ : Virtual angioscopy post-processing technique is used to visualize the coronary Ostia (yellow arrowhead). The yellow arrow points to the origin of the right coronary artery. $\mathrm{LM}=$ Left main coronary artery. $\mathrm{RCS}=$ Right coronary sinus of Valsalva.

\section{Figure 2:}

A: Two-dimensional transoesophageal echocardiography in mid-oesophageal short-axis view of the aortic valve reveals the anomalous origin of the left coronary artery from the opposite sinus with an inter-arterial course. The right and left coronary arteries shared the same coronary ostium. The anomalous coronary artery had an acute take-off angle (defined as $<45 \mathrm{deg}$ ) with an origin below the commissure. B: Color flow Doppler demonstrates flow through the left anomalous coronary artery. C-D: We used three-dimensional transoesophageal echocardiography to allow the observer to look directly at the ostium of the coronary artery. First, mid-oesophageal aortic valve short axis view was obtained rotating the transducer angle to 30deg. Slight anteflexion of the probe was required. Once a wide-angled acquisition of the three-dimensional data set of the aortic root was obtained, the cropping plane was aligned parallel to the coronary artery ostium. The origin of the right coronary artery can be seen (yellow arrow) inside the shared coronary ostium (yellow arrowhead). LM = Left main coronary artery. RCS = Right coronary sinus of Valsalva.

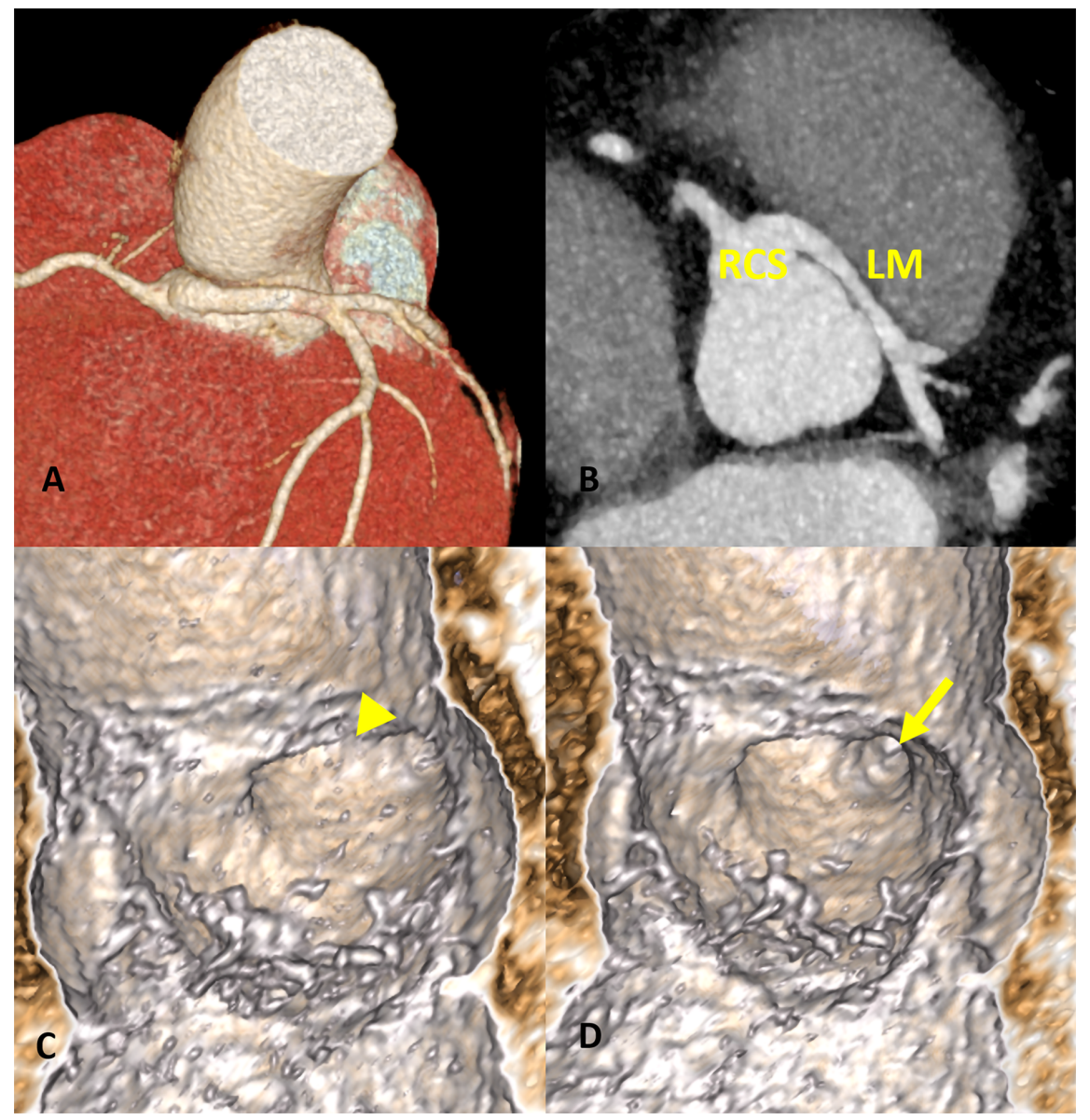




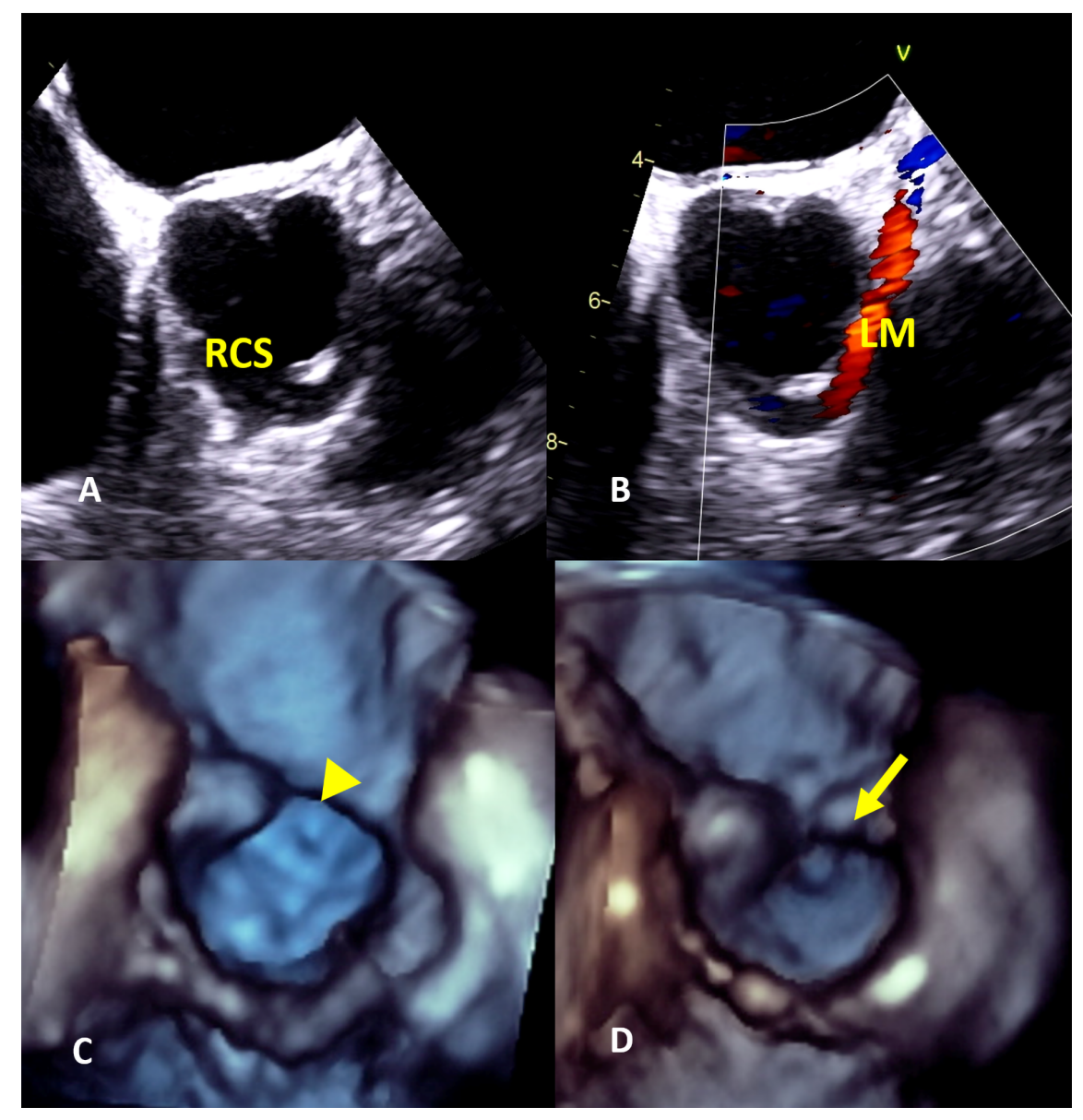

